\title{
Antifoams: the overlooked additive?
}

\section{Sarah J Routledge*,1, David R Poyner1 \& Roslyn M Bill1}

It is well known that foaming can become a major problem during large-scale recombinant protein bioprocesses; the vigorous stirring and introduction of gases to maintain the required dissolved oxygen (DO) concentration for the organism [1] in addition to the growth medium and protein products themselves [2], can result in the formation and stabilization of foams consisting of gas-filled liquid lamellas. Both unstable high liquid content foams and stable dry polyhedric foams may be present in a bioprocess [3] and readily accumulate. Unchecked build up of foams may result in foam escaping from the vessel, loss of sterility [4] and material, while bursting bubbles may damage proteins and cells [5] in addition to the blockage of exit filters which can pressurize the vessel and damage equipment.

A common method of foam destruction is the addition of chemical antifoaming agents. Many types of antifoams are commercially available with a range of properties and varied foam destruction efficiency, with some becoming depleted requiring several additions over time [6]. They may contain surfactants and can consist of hydrophobic solids dispersed in carrier oil, aqueous suspensions or emulsions, liquid single components or solids [7]. Some examples of antifoams of a range of types are; Antifoams A and C (Sigma) which are both $30 \%$ emulsions of silicone polymer, J673A (Struktol) an alkoxylated fatty acid ester on a vegetable base, P2000 (Fluka) which is a polypropylene glycol, PEG600 and PEG8000 (Sigma) which are both polyethylene glycols, S184 (Wacker-Chemie Co.) which is a silicone oil, SB2121 (Struktol) a polyalkylene glycol, SE9 (Wacker-Chemie Co.) an emulsion containing 10\% S184, and SLM54474 (Wacker-Chemie Co.) a polypropylene glycol. Most investigations concerning antifoams evaluate their defoaming capabilities; foam formation has been thoroughly characterized, and the main mechanisms of foam destruction have been explained, although some details of their action are not completely understood. This is partly due to submicroscopic events occurring during foam destruction and the complex involvement of the numerous active components of the agents [6], about which little information is generally provided by the manufacturer. Methods such as the Bartsch shaking test [8] and Ross-Miles pouring test [9] allow simple evaluation of antifoam efficiency and mathematical models have been generated allowing optimization of their addition to bioprocesses [6].

Unexpectedly, considering their frequent use as an additive, there is significantly less information available concerning the biological effects of antifoaming agents and very little of this is recent. Present research has found that antifoams can have a broad range of effects upon bioprocesses, both on the culture environment and upon the cells themselves. The effects of these agents therefore warrant a more detailed evaluation. However, while many studies producing recombinant protein by large scale fermentation report the addition of antifoams, usually only the volume or concentration of the agent used is stated; the volumes added seem to be arbitrary and no rationalization for the type and concentration used is provided. Furthermore, it is usually unclear whether several antifoams have been evaluated previously by the authors but not reported. In general, antifoams are apparently added without regard to their potential biological impact upon the process.

The effect of antifoams upon oxygen transfer rate (OTR) and the volumetric mass oxygen transfer coefficient (kLa), upon which OTR is dependent, is well studied. Their effects are varied, depending upon the type and concentration of antifoam and may significantly increase or reduce the OTR $[10,11]$, which could in turn affect the DO availability in the process. However, information regarding the effects of antifoams upon oxygenation of living cultures is extremely limited, and it is unclear how much of an impact the antifoams and concentrations commonly used are exerting. Koch et al found that silicone oil-containing antifoams affected the OTR at the beginning of a process, but the effects reduced throughout [12]. In contrast, our findings for five different antifoams demonstrated that although kLa was significantly affected for most of the concentrations investigated, it did not appear to affect the DO levels in the culture [11]. In aerobic bioprocesses, a high OTR is desired to maintain growth as oxygen has a low 
solubility in water. A low OTR could lead to anaerobic respiration and the production of ethanol or acetic acid [13], which could inhibit growth. Certain recombinant proteins may be sensitive to DO, such as the proprotein penicillin acylase which requires oxygen for maturation [13]. It is therefore important to evaluate the effects of each individual antifoam and concentration to be used as the effects are diverse and not well documented.

Antifoams have been found to have both positive and negative effects upon cell growth. This could potentially influence the volumetric yields of protein produced and positive effects could be beneficial in particular to the production of membrane proteins. Growth defects to Schizosaccharomyces pombe cultures have been observed with 1\% v/v PEG8000 [14]. Specific growth rates of Escherichia coli K12 cells were found to be decreased by specific concentrations of PPG/silicone antifoam and increasing PPG antifoam concentration, but higher growth rates were observed with an antifoam emulsion [12]. Concentrations of up to $8 \% \mathrm{v} / \mathrm{v} \mathrm{J673A}$ increased the optical density of Pichia pastoris cells whereas concentrations of up to $8 \%$ v/v of SB2121 added to Saccharomyces cerevisiae cultures decreased optical density, while Antifoam $\mathrm{C}$ had no effect. The antifoams also appeared to interact differently with cultures depending upon the medium [5]. We observed that antifoams P2000 and SB2121 are able to increase the density of P. pastoris cultures, and upon further investigation found that J673A, P2000 and SB2121 containing cultures had higher growth rates than those without antifoam [15]. A recent study investigated the effects of various factors including addition of a silicone-based antifoam upon P. pastoris producing $\beta$-glucosidase I (BGLI) in baffled and non-baffled shake flasks. The authors found an improvement to cell growth in the presence of the antifoam, although there was no enhancement to the enzyme activity [16].

There is evidence to suggest that antifoams can alter the morphology of cells and interact with cell walls of prokaryotes [17]. Our findings suggest that antifoams can alter the permeability of yeast plasma membranes, which resulted in enhanced green fluorescent protein secretion [11]. These findings agree with a study suggesting that antifoams could alter sterol biosynthesis leading to changes in permeability [18], and is also in agreement with our preliminary electrospray mass spectrometry findings where we observed changes to relative phosphatidylcholine composition in 1\% P2000 culture samples, in addition to each antifoam-containing sample having changes to relative phosphatidylinositol composition [Routledge SJ, Unpublished Data].

It is important to ensure that the antifoams added to a process are not toxic to the cells [6] and at what concentration, if any, toxicity occurs. Upon propidium iodide staining of antifoam-containing cultures, we did not observe any detrimental effects to the viability of the cells as measured by flow cytometry at the concentrations of up to $1 \% \mathrm{v} / \mathrm{v}$ antifoam investigated [11]. There is a surprising lack of information in this particular aspect of antifoam use, and the effects of antifoams upon viability had not previously been reported suggesting that this is not considered routinely.

Due to their range of possible interactions with various aspects of bioprocesses, antifoams can therefore influence recombinant protein production yields in prokaryotic and eukaryotic expression systems. Certain antifoams and concentrations appear to decrease yields, while others can significantly improve them. Studies have shown that increasing concentrations of PPG and PPG/silicone antifoams improved both volumetric and specific product activity of $\beta$-galactosidase fusion protein produced by $\mathrm{E}$. coli K12, while silicone oil decreased it [12]. We found that the yield of secreted green fluorescent protein produced by $\mathrm{P}$. pastoris in shake flasks increased at concentrations of at least $0.4 \% \mathrm{v} / \mathrm{v}$ for Antifoam A, Antifoam C, while addition of 1\% v/v J673A, 1\% v/v P2000 and 1\% v/v SB2121 almost doubled the yield [11]. We have also observed a reversal of concentration effect with production of the human adenosine 2a receptor (hA2aR), a GPCR, and upon scale up into 2 I bioreactors [Routledge SJ, Unpublished Data]. Holmes et al observed that yields of a recombinant Fc fusion protein expressed by $\mathrm{P}$. pastoris and $\mathrm{S}$. cerevisiae were decreased when concentrations above $1 \% \mathrm{v} / \mathrm{v}$ of J673A, SB2121 and Antifoam C were added [5]. Another study showed that a combination of PEG600 and PEG3350 could double $\alpha$ amylase production in Bacillus species. The same study also demonstrated that particular concentrations of the same antifoam could improve yields for Bacillus subtillis but reduce it for Bacillus amyloliquefaciens [17]. These findings highlight the fact that antifoam effects can 
vary hugely with each individual set up, and should therefore be evaluated in each process; a particular type and concentration that works effectively in one process could be detrimental to productivity in another.

It is likely that antifoams affect a combination of factors during bioprocesses. It can therefore be difficult to predict the outcome upon cell growth and recombinant protein production without further investigation. At present, antifoams are rarely specifically evaluated as an additive despite their documented effects. Recently, a study investigated the effects of a silicone-based antifoam upon shake flask P. pastoris cultures. While the concentration of the antifoam used was not stated and just one type was tested, it is encouraging that informative data on antifoams had been included in the study [16]. In order to reduce or take advantage of the effects of antifoams for optimized processes, trials should be conducted with a panel of antifoams routinely used, including several different types at a range of concentrations. Simple tests can determine the key factors influenced by antifoams; efficiency of foam destruction, viability and effect upon KLa, DO and ultimately their effect upon cell growth and protein expression [19]. The suitability of each agent should be evaluated on a case by case basis with regard to their effects. In addition, certain antifoams are not US FDA approved and others may coat equipment or damage membranes during downstream processing [20]. Considering the vast number, range of types and concentrations that are routinely added to reduce foaming coupled with the possible interaction of antifoams within bioprocesses, any information obtained from future studies will greatly expand current knowledge and understanding of these commonly used agents.

\section{References}

1. Etoc A, Delvigne F, Lecomte JP, Thonart P. Foam Control in Fermentation Bioprocess: From simple aeration tests to bioreactor. Appl. Biochem. Biotechnol. 129-132, 392-404 (2006).

2. Christiano SP, Fey KC. Silicone antifoam performance enhancement by nonionic surfactants in potato medium. J. Ind. Microbiol. Biotechnol. 30, 13-21 (2003).

3. Koller K. Foam control in fermentation processes: while chemical defoamers are ideal for foam prevention, mechanical systems provide gentle defoaming through a combination of foam destruction and foam recycling. Chemical Engineering, 1 August (2004).

4. Varley J, Brown A, Boyd R, Dodd P, Gallagher S. Dynamic multipoint measurement of foam behaviour for a continuous fermentation over a range of key process variables. Biochem. Eng. J. 20, 61-72 (2004).

5. Holmes W, Smith R, Bill R. Evaluation of antifoams in the expression of a recombinant FC fusion protein in shake flask cultures of Saccharomyces cerevisiae. Microb. Cell Fact. 5, 30 (2006).

6. Pelton R, Flaherty T. Defoamers: linking fundamentals to formulations. Polym. Int. 52, 469-485 (2003).

7. Höfer R. Struktol Foams and Foam Control. (2008).

8. Bartsch O. Über Schaumsysteme (1942).

9. Rosen J, Solash J. Factors affecting initial foam height in the Ross-Miles foam test. J. Am. Oil Chem. Soc. 46, 399-402 (1968).

10. Morao A, Maia C, Fonseca M, Vasconcelos J, Alves S. Effect of antifoam addition in gas-liquid mass transfer in stirred fermenters. Bioprocess Engineering 20, 165-172 (1999).

11. Routledge SJ, Hewitt CJ, Bora N, Bill RM. Antifoam addition to shake flask cultures of recombinant Pichia pastoris increases yield. Microb. Cell Fact. 10(17), 1-11 (2011).

12. Koch V, Rüffer H, Schügerl K, Innertsberger E, Menzel H, Weis J. Effect of antifoam agents on the medium and microbial cell properties and process performance in small and large reactors. Process Biochemistry 30, 435-446 (1995).

13. Palomares LA, Estrada-Mondaca S, Ramírez OT. Production of recombinant proteins challenges and solutions. Methods Mol. Biol. 2, 15-51 (2004).

14. Mukaiyama H, Giga-Hama Y, Tohda H, Takegawa K. Dextran sodium sulphate enhances secretion of recombinant human transferrin in Schizosaccharomyces pombe. Appl. Microbiol. Biotechnol. 85, 155-164 (2009).

15. Routledge SJ. Beyond de-foaming: the effects of antifoams on bioprocess productivity. Comput. Struct. Biotechnol. J. 3(4), e201210014 (2012).

16. Batra J, Beri D, Mishra S. Response surface methodology based optimization of $\beta$-glucosidase production from Pichia pastoris. Appl. Biochem. Biotechnol. 172(1), 380-393 (2013).

17. Andersson E, Ramgren M, Hahn-Hagerdal B. The Influence of PEG on $\alpha$-amylase production with Bacillus species. Biochemical Engineering $506,613-616$ (1987).

18. Pawiroharsono S, Naji B, Bonaly R, Tonetti F, Chasseboeuf C, Richter P. Permeability and membrane sterol distribution in Saccharomyces uvarum and Kluyveromyces bulgaricus grown in presence of polyoxyalkylene lycol-oleic acid condensates. Appl. Microbiol. Biotechnol. 27, 181-185 (1987).

19. Routledge SJ, Bill RM. The effect of antifoam addition on protein production yields. In: Recombinant Protein Production in Yeast, Bill RM. Humana Press, NY, USA, 87-97 (2012).

20. Mcgregor WC, Weaver JF, Tansey SP. Antifoam effects on ultrafiltration. Biotechnol. Bioeng. 31(4), 385-389 (2004). 